\title{
Introduction to mammalian genome special issue: the microbiome in human health and disease
}

\author{
Guillaume Pavlovic ${ }^{1}$ · Je Kyung Seong ${ }^{2,3,4}$. George M. Weinstock ${ }^{5,6}$
}

Accepted: 21 June 2021 / Published online: 30 June 2021

(c) The Author(s), under exclusive licence to Springer Science+Business Media, LLC, part of Springer Nature 2021

The impact of microorganisms and viruses on human health is evident to all of us. Today, the pandemic of the COVID-19 virus, which has completely changed the life of the whole planet, can only remind us of this fact. But the interactions between microorganisms and their hosts are not limited to infections by pathogens. The importance of the human microbiota, including the role of commensal microorganisms, is increasingly being emphasized. In the last 10 years, thanks to advances in technology, discoveries in this field have accelerated, both in humans and in animal models such as the mouse. Striking examples of the much broader role of microbiota are accumulating. Influences of the microbiome on the biology and diseases of virtually all parts of the body, from the gut to the brain, are now documented. It is now necessary to consider the microbiome in order to get a complete picture of underlying mechanisms.

With this perspective in mind, in this issue we present forays into a variety of aspects of microbiome biology. Ryu et al. review how the microbiota is associated with human disease and contributes to drug responses, and Kim et al.

Guillaume Pavlovic

pavlovic@igbmc.fr

1 PHENOMIN-Institut Clinique de la Souris, CELPHEDIA, CNRS, INSERM, Université de Strasbourg, Illkirch-Graffenstaden, 67404 Strasbourg, France

2 Lab. of Developmental Biology and Genomics, Research Institute for Veterinary Science, and BK 21 PLUS Program for Creative Veterinary Science Research, College of Veterinary Medicine, Seoul National University, Seoul 08826, South Korea

3 Interdisciplinary Program for Bioinformatics, Program for Cancer Biology and BIO-MAX/N-Bio Institute, Seoul National University, Seoul 08826, South Korea

4 Korea Mouse Phenotyping Center (KMPC), Seoul National University, Seoul 08826, South Korea

5 Jackson Laboratory for Genomic Medicine, Farmington, CT 06032, USA

6 UConn Health, Farmington, CT 06032, USA focus on the role of gut microbiota in the immune checkpoint. Although it represents the largest part of the human microbiotas, the intestinal microbiota is not the only microorganism environment. Brubaker et al. illustrate this with a review on the importance of the urobiome, the microbiome of the urinary bladder.

In animal models, the understanding of the factors that impact the genome-phenotype relationship is a major challenge to guarantee the reproducibility of the experiments. Ericsson et al. highlight the influence of the gut microbiota on phenotype data and reproducibility in the mouse. Likewise, Hansen et al. outline the role of the gut microbiota in modeling human disease in rodents. The review by Bubier et al. highlights the influence of host genetics on microbial composition.

A final question tackled in this issue is how to obtain microbiota data and ensure their relevance. Yen et al. present current bioinformatics strategies for handling metagenomic sequence data and Yang et al. describe the methodologies that can be used to determine the gut bacterial profile in laboratory mice. Finally, Kwon et al. present how the mouse model can better mimic human physiology through the contribution of wild mice and their microbiota.

Research on the microbiota is advancing rapidly and new perspectives appear frequently. In particular, knowledge and management of technological and environmental biases and limitations are increasingly being defined. The discovery of individual species and the function of their genes is progressing. Correlations between the microbiome and various diseases are increasingly apparent and clinical trials are ongoing in humans, using microbiome therapy. We are at the beginning of this journey, scarcely a decade into the modern microbiome era, and the outlook is promising and enormous.

Publisher's Note Springer Nature remains neutral with regard to jurisdictional claims in published maps and institutional affiliations. 